\title{
Avant propos : Revisiter la Grande Guerre
}

Foreword: Revisiting the Great War

\section{Florence Binard et John Mullen}

\section{OpenEdition}

\section{Journals}

Édition électronique

URL : http://journals.openedition.org/rfcb/242

DOI : $10.4000 / \mathrm{rfcb} .242$

ISSN : 2429-4373

Éditeur

CRECIB - Centre de recherche et d'études en civilisation britannique

Édition imprimée

Date de publication : 15 janvier 2015

ISSN : 0248-9015

Référence électronique

Florence Binard et John Mullen, « Avant propos : Revisiter la Grande Guerre », Revue Française de Civilisation Britannique [En ligne], XX-1 | 2015, mis en ligne le 01 mai 2015, consulté le 04 mai 2019. URL : http://journals.openedition.org/rfcb/242 ; DOI : 10.4000/rfcb.242

Ce document a été généré automatiquement le 4 mai 2019.

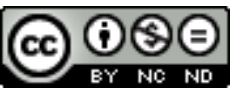

Revue française de civilisation britannique est mis à disposition selon les termes de la licence Creative Commons Attribution - Pas d'Utilisation Commerciale - Pas de Modification 4.0 International. 


\section{Avant propos : Revisiter la Grande Guerre}

Foreword: Revisiting the Great War

Florence Binard et John Mullen

\section{NOTE DE L'ÉDITEUR}

Remerciements pour table ronde :

Nous remercions chaleureusement William Philpott, Neil Faulkner, Pat Thane et Ana

Carden Coyen d'avoir accepté de se livrer à cet exercice de « table ronde écrite ».

1 En cette période d'effervescence médiatique et culturelle autour des commémorations du centenaire de la Première Guerre mondiale, il nous a semblé qu'un numéro sur cette période s'imposait et ce d'autant plus que le sujet n'avait jamais été traité dans notre revue.

2 Les perceptions, les interprétations que l'on fait de l'histoire sont sans cesse renouvelées à l'aune de nos connaissances mais également en fonction de nos subjectivités présentes. Ainsi, les visions de ce qu'on a appelé « la Grande Guerre » ou « la der des ders » se sont vues modifier au fil des décennies en raison des événements qui ont jalonné et marqué le vingtième siècle, mais également du fait de l'évolution de la société et des modes de pensée. Si le sens donné au centenaire est indissociable de l'histoire dans laquelle il est ancré, il est surtout le produit de notre époque et mérite qu'on l'étudie. Revisiter le passé participe de la fabrique de l'histoire.

3 La première partie de ce numéro est consacrée à l'historiographie récente et aux débats contradictoires soulevés par l'ampleur des commémorations, l'objectif étant d'apporter à nos lecteurs des clés leur permettant de mieux appréhender les productions pléthoriques et les controverses complexes qui se sont d'ores et déjà fait jour et qui continueront, sans aucun doute, d'agiter des débats au Royaume Uni jusqu'en 2018. 
4 Le premier article est une présentation synthétique, par John Mullen, des multiples manifestations de commémoration qui se sont déroulées en Grande-Bretagne en 2014. Allant des cérémonies officielles célébrées en grande pompe à la fabrication de bières commémoratives, en passant par la création de spectacles musicaux antimilitaristes, sans oublier l'omniprésent coquelicot en papier que portent de nombreux Britanniques au mois de novembre, ce panorama de la diversité des actions menées - illustré, en annexe, par une série de photos des actions mentionnées - permet de mieux comprendre le contexte des débats et d'en saisir la complexité. En effet, il en ressort que le consensus apparent sur certains aspects de la commémoration se révèle parfois de surface.

Vient ensuite une sorte de "table-ronde écrite» dans laquelle quatre historien-ne-s britanniques, d'horizons très différents les uns des autres, nous livrent leurs impressions sur l'état de l'historiographie dans leur domaine de spécialité respectif ainsi que leurs points de vue sur le rôle des historien-ne-s durant ces années de commémoration. Le premier des auteurs est William Philpott, professeur d'histoire à King's College London et spécialiste de l'histoire militaire. Son ouvrage, Bloody Victory $(2010)^{1}$ a largement contribué à la défense des capacités militaires des généraux anglais d'il y a un siècle. Dans ce livre, Philpott souligne le processus d'apprentissage ("learning curve») des responsables militaires et dénonce leur réputation d'incapables, réputation non méritée, selon lui, mais qui néanmoins perdure à ce jour. Dans son article pour ce numéro, M. Philpott s'intéresse aux évolutions récentes de l'histoire militaire de la Grande Guerre et note avec satisfaction que l'histoire des batailles rencontre un intérêt croissant depuis les années 1990. Il souligne, par ailleurs, l'importance de la nécessaire collaboration entre l'armée britannique et l'armée française et conclut sur la pertinence d'une recherche historique fondée sur une approche comparative transnationale.

6 Neil Faulkner, membre du comité de rédaction de la revue Current Archaeology et auteur d'ouvrages sur l'antiquité romaine, est le deuxième contributeur pour cette «table ronde ». Il représente, d'une part, la discipline, relativement jeune, d'archéologie de la Première Guerre mais, également, d'autre part, une historiographie militante en opposition avec le discours dominant sur la guerre. Membre dirigeant de la campagne " No Glory in War », il est de ces historien-en-s sociaux qui contestent le récit officiel d'une guerre juste et héroïque.

7 Pat Thane, professeure d'histoire contemporaine à King's College London, et élue à la British Academy, s'interroge sur les raisons de l'extraordinaire popularité des commémorations du centenaire de la Première Guerre mondiale en Grande-Bretagne. Elle nous propose un bref panorama des manifestations organisées dans plusieurs pays dont l'Allemagne, l'Irlande, les Etats Unis ou encore l'Australie et pointe le caractère national voire nationaliste du choix des événements commémorés.

8 Enfin, Ana Carden Coyen, co-directrice du Centre for the Cultural History of War à l'Université de Manchester, dont le dernier ouvrage traite des politiques médicales durant la Grande Guerre ${ }^{2}$, présente une riche historiographie des études de genre et de l'histoire de la sexualité en lien avec la Première Guerre mondiale. Son intérêt porte principalement sur les masculinités, c'est-à-dire sur l'adhésion ou la désobéissance des soldats aux normes masculines de leur temps mais également sur les perceptions et constructions de ces normes, sans omettre l'aspect « racial » de ces questions. Ana Carden Coyen conclut sur l'importance de la participation des historien-ne-s à l'élaboration du contenu des manifestations de commémoration qui ont tendance à être centrées sur «l'homme blanc » et sur l'Europe. 

du passé. consacrés aux témoignages individuels. Estèves, n'a pas été appréhendée à sa juste valeur. portant sur la Première Guerre mondiale.

Deux autres articles complètent la section historiographique.

Marc Calvini-Lefebvre, maître de conférences à l'Université d'Aix-Marseille, traite la question des controverses féministes autour de l'histoire de la Grande Guerre. Il explore les trois 'configurations' qui, selon lui, ont marqué l'étude de la Grande Guerre dans l'histoire du féminisme britannique. Il explique comment chacune a été caractérisée par ses propres questions, fruits du contexte politique du moment. Surtout, il montre combien le «standpoint », le lieu d'où parle l'historien-ne, influence et oriente sa lecture

Enfin, John Mullen nous livre une analyse des différents tournants historiographiques concernant la Première Guerre mondiale. Jusqu'aux débuts des années 1960, le point de vue de l'histoire militaire et diplomatique domine. Il s'ouvre alors, dans le milieu académique, du moins, à l'histoire sociale et, ensuite, à l'histoire culturelle. Une étude détaillée de la centaine de thèses sur la Première Guerre mondiale soutenues dans les universités du Royaume Uni depuis 2000 montre, en effet, qu'il existe un contraste entre les thèmes de prédilection étudiés dans les universités et les succès d'édition, largement

Une deuxième section de notre numéro s'intéresse à la place des femmes dans la guerre.

Katherine Connolly, auteure d'une biographie de Sylvia Pankhurst ${ }^{3}$, nous éclaire sur le militantisme de cette fille d'Emmeline Pankhurst qui, comme sa mère, fit campagne pour le droit de vote des femmes mais qui, contrairement à sa mère, très patriotique, s'est investie dans un combat pacifiste et socialiste pour une société dans laquelle les femmes de la classe ouvrière seraient leurs propres représentantes. Katherine Connolly retrace les idées politiques de Sylvia Pankhurst entre 1914 et 1918 et montre la cohérence de son cheminement intellectuel allant de la défense du suffrage universel dans le cadre de la démocratie parlementaire à celle du modèle de la démocratie soviétique.

Sylvie Pomiès-Maréchal, maîtresse de conférences à l'université d'Orléans, nous propose une analyse des récits de l'expérience d'infirmières britanniques, parties en France soigner les blessés. Si les écrits étudiés sont de nature très différente, allant du journal intime à l'ouvrage de propagande patriotique commissionné par le Scottish Women's Hospital et publié au beau milieu de la guerre, en 1916, Sylvie Pomiès-Maréchal nous montre qu'ils se rejoignent sur la question de la perception des rôles attribués à chaque sexe et convergent quant à leur adhésion aux normes de genre.

Les deux articles de la troisième section sont des illustrations de l'immense variété possible d'objets d'étude sur la Première Guerre mondiale.

Henry Daniels étudie le rôle de l'industrie du tabac et ses diverses méthodes de mise à profit de la situation de guerre pour renforcer sa position économique. Il s'intéresse notamment aux campagnes publicitaires et aux images genrées qu'elles véhiculaient: masculinité et virilité pour les hommes, émancipation et séduction pour les femmes.

Olivier Estèves nous livre son analyse de l'évolution des écrits de Bertrand Russell durant la guerre. Adoré et haï à l'époque à cause de ses prises de position contre la guerre, Russell développa une vision complexe de la guerre et des guerres qui, selon Olivier

Les trois comptes rendus de lecture inclus dans ce numéro concernent des ouvrages 
Florence Binard nous invite à découvrir Disturbing Practices History, Sexuality and Women's Experience of Modern War (2013) de Laura Doan. Susan Finding présente l'ouvrage de John Mullen, The Show Must Go On: la Chanson Populaire en Grande Bretagne pendant la Grande Guerre, et enfin, Jean-Louis Legalery nous propose sa lecture du livre de Will Ellsworth-Jones, We Will Not Fight...The Untold Story of World War One's Conscientious Objectors.

\section{NOTES}

1. William Philpott, Bloody Victory: The Sacrifice on the Somme and the Making of the Twentieth Century, Londres, Abacus, 2010.

2. Ana Carden Coyne, The Politics of Wounds: Military Patients and Medical Power in the First World War, Oxford,Oxford University Press, 2014.

3. Katherine Connolly, Sylvia Pankhurst: Suffragette, Socialist and Scourge of Empire, Londres, Pluto Press, 2013.

\section{RÉSUMÉS}

En cette période d'effervescence médiatique et culturelle autour des commémorations du centenaire de la Première Guerre mondiale, il nous a semblé qu'un numéro sur cette période s'imposait et ce d'autant plus que le sujet n'avait jamais été traité dans notre revue. Nous avons choisi de consacrer la plus grande partie de la revue à des questions historiographiques, plutôt que de se concentrer sur un aspect spécifique de la période de guerre.

The tremendous number of publications and events surrounding the centenary of the First World War made this an obvious choice for an issue of our journal, even more so since the Revue française de civilisation britannique has not yet dealt with this event. Given the huge number of historical writings about this conflict, we have chosen to consecrate mushc of the issue to historiographical questions, rather than concentrate on a single aspect of the war.

\section{INDEX}

Keywords : British history, First World War, historiography, centenary

Mots-clés : Histoire britannique, Première Guerre mondiale, historiographie, centenaire 
AUTEURS

FLORENCE BINARD

Université Paris Diderot

JOHN MULLEN

Université Paris-Est Créteil 